ISSN: $1311-1728$ (printed version); ISSN: 1314-8060 (on-line version) doi: http://dx.doi.org/10.12732/ijam.v26i2.2

\title{
CONVECTION HEAT AND MASS TRANSFER IN
}

\section{A HYDROMAGNETIC FLOW OF A MICROPOLAR FLUID OVER A POROUS MEDIUM}

\author{
B.I. Olajuwon ${ }^{1}$, J.I. Oahimire ${ }^{2}$, M.A. Waheed ${ }^{3}$ \\ ${ }^{1}$ Department of Mathematics \\ Federal University of Agriculture \\ Abeokuta, NIGERIA \\ ${ }^{2}$ Department of Mathematics \\ University of Port Harcourt \\ Port Harcourt, NIGERIA \\ ${ }^{3}$ Department of Mechanical Engineering \\ Federal University of Agriculture \\ Abeokuta, NIGERIA
}

\begin{abstract}
This study presents a mathematical analysis of a hydromagnetic boundary layer flow, heat and mass transfer characteristics on steady twodimensional flow of a micropolar fluid over a stretching sheet embedded in a non-Darcian porous medium with uniform magnetic field in the presence of thermal radiation. The governing system of partial differential equations is first transformed into a system of non-linear ordinary differential equation using the usual similarity transformation. The resulting coupled non-linear ordinary differential equations are then solved using perturbation technique. With the help of graphs, the effects of the various important parameters entering into the problem on the velocity, temperature and concentration fields within the boundary layer are separately discussed. The effects of the pertinent parameters on the
\end{abstract}

Received: December 11, 2012

(C) 2013 Academic Publications

${ }^{\S}$ Correspondence author 
wall temperature, wall solutal concentration, skin friction coefficient and the rate of heat and mass transfer are presented numerically in tabular form. The results obtained showed that these parameters have significant influence on the flow.

AMS Subject Classification: 76Wxx, 76Dxx

Key Words: mass transfer, hydromagnetic flow, porous medium, perturbation technique, heat transfer

\section{Introduction}

The study of heat and mass transfer has attracted the interest of numerous researchers due to its applications in sciences and engineering problems. Such applications include nuclear reactor, MHD generators, geothermal energy extractions, analyzing the behaviour of exotic lubricant, the flow of colloidal suspension or polymeric fluid and the boundary layer controlling the field of aerodynamics. In nature and industrial applications many transport processes exist where the transfer of heat and mass takes place simultaneously as a result of combined buoyancy effects of thermal diffusion and diffusion of chemical species. In chemical process industries such as food processing and polymer production, the phenomenon of heat and mass transfer is also encountered. Rebhi [12] studied unsteady natural convection heat and mass transfer of micropolar fluid over a vertical surface with constant heat flux. The governing equations were solved numerically using McCormack's technique and effects of various parameters were investigated on the flow. Eldabe and Ouaf [5] solved the problem of heat and mass transfer in a hydromagnetic flow of a micropolar fluid past a stretching surface with ohmic heating and viscous dissipation using the Chebyshev finite difference method. Keelson and Desseaux [6] studied the effect of surface conditions on the flow of a micropolar fluid driven by a porous stretching surface. The governing equations were solved numerically. Sunil et al. [15] studied the effect of rotation on a layer of micropolar ferromagnetic fluid heated from below saturating a porous medium. The resulted non-linear coupled differential equations from the transformation were solved using finite-difference method. Rahman and Sultan [13] studied the thermal radiation interaction of the boundary layer flow of micropolar fluid past a heated vertical porous plate embedded in a porous medium with variable suction as well as heat flux at the plate. The governing equations were solved numerically by an efficient, iterative, finite - difference method. Mahmoud [7] investigated thermal radiation effect on magneto hydrodynamic flow of a mi- 
cropolar fluid over a stretching surface with variable thermal conductivity. The solution was obtained numerically by iterative, Runge-Kuta order-four method. Magdy [11] studied unsteady free convection flow of an incompressible electrically conducting micropolar fluid, bounded by an infinite vertical plane surface of constant temperature with thermal relaxation including heat sources. The governing equations were solved using Laplace transformation. The inversion of the Laplace transforms was carried out with a numerical method. Mohammed and Abo-Dahah [8] investigated the effects of chemical reaction and thermal radiation on heat and mass transfer in magneto hydrodynamic micropolar flow over a vertical moving porous plate in a porous medium with heat generation. The solution was obtained numerically by finite-difference method. Bayomi et al. [2] consider magneto hydrodynamic flow of a micropolar fluid along a vertical semi-infinite permeable plate in the presence of wall suction or injection effects and heat generation or absorption. The obtained self-similar equation were solved numerically by an efficient implicit, iterative, infinite-difference method. Reena and Rana [14] investigated double-diffusive convection in a micropolar fluid layer heated and soluted from below saturating a porous medium. A linear stability analysis theory and normal mode analysis method was used. Mohammed et al [9] studied magneto hydrodynamic convection with thermal radiation and mass transfer of micropolar fluid through a porous medium occupying a semi-infinite region of the space bounded by an infinite vertical porous plate with constant suction velocity in the presence of chemical reaction, internal heat source, viscous and Darcy's dissipation. The highly non-linear coupled differential equations governing the boundary layer flow, heat and mass transfer were solved using finite difference method. Heat and mass transfer in a hydromagnetic flow have many applications in science and engineering. This present model have applications in biomedical and engineering. For instance in the dialysis of blood in artificial kidney, flow in oxygenation, etc. Engineering applications includes the porous pipe design, design of filter, etc. Motivated by the above previous works and possibly applications, the present paper study heat and mass transfer in a hydromagnetic flow of a micropolar fluid over a porous medium using Boussineq model in the presence of uniform magnetic field. The transformed non-linear boundary layer equations together with the boundary conditions are solved analytically using perturbation technique. 


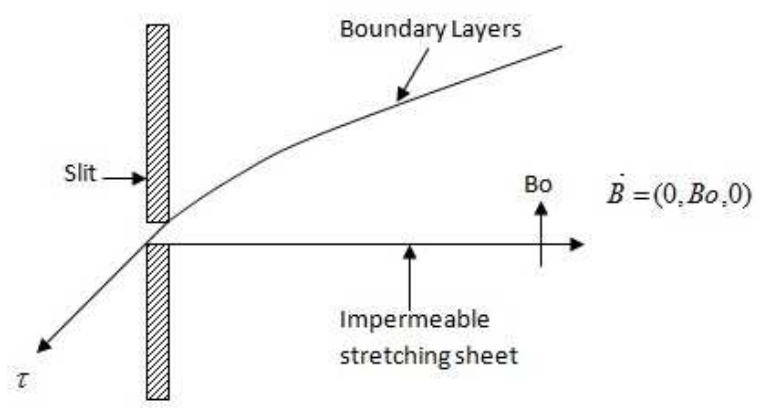

Figure 1: Physical model

\section{Mathematical Formulation}

We consider a steady, two-dimensional mixed convection flow of an incompressible, electrically conducting micropolar fluid over a stretching sheet. The fluid flows towards a surface coinciding with the plane $y=0$, the flow region $(y>0)$. The origin is fixed as shown in Figure 1. The $x$-axis is taken in the direction along the sheet and $y$-axis is taken perpendicular to it. The flow is generated by the action of two equal and opposite forces along the $x$-axis and the sheet is stretch in such a way that the velocity at any instant is proportional to the distance from the origin $(x=0)$. The flow field is exposed to the influence of an external transverse magnetic field of strength $\bar{B}=\left(0, B_{o}, 0\right)$.

With these assumptions, the continuity equation, momentum equation, angular momentum equation, energy equation and mass diffusion equation governing the flow are

$$
\begin{aligned}
& \frac{\partial u}{\partial x}+\frac{\partial v}{\partial y}=0 \\
& u \frac{\partial u}{\partial x}+v \frac{\partial u}{\partial y}=\left(\nu+\frac{k_{1}^{*}}{\rho}\right) \frac{\partial^{2} u}{\partial y^{2}}+\frac{k_{1}^{*}}{\rho} \frac{\partial N}{\partial y}-\frac{\nu \varphi}{k} u-\frac{C_{b} \varphi}{\sqrt{k}} u^{2}-\frac{\sigma}{\rho} B_{0}^{2} u \\
& +g \beta_{t}\left(T-T_{\infty}\right)+g \beta_{c}\left(C-C_{\infty}\right), \\
& \rho j\left(u \frac{\partial u}{\partial x}+v \frac{\partial N}{\partial y}\right)=\gamma \frac{\partial^{2} N}{\partial y^{2}}-k_{1}^{*}\left(2 N+\frac{\partial u}{\partial y}\right), \\
& u \frac{\partial T}{\partial x}+v \frac{\partial T}{\partial y}=\frac{\kappa}{\rho C_{p}} \frac{\partial^{2} T}{\partial y^{2}}-\frac{1}{\rho C_{p}} \frac{\partial q_{r}}{\partial y}+\frac{\sigma}{\rho C_{p}} B_{0}^{2} u^{2}+\frac{q^{\prime \prime \prime}}{\rho C_{P}}+\frac{\mu}{\rho C_{P}}\left(\frac{\partial u}{\partial y}\right)^{2},
\end{aligned}
$$




$$
u \frac{\partial C}{\partial x}+v \frac{\partial C}{\partial y}=D \frac{\partial^{2} C}{\partial y^{2}}
$$

Here $u$ and $v$ are the velocity components along $x$ and $y$ directions, $\rho$ is the density, $T$ is the temperature of the fluid, $C_{b}$ is the form of drag coefficient which is independent of viscosity and other properties of the fluid but is dependent on geometry of the medium, $k$ is permeably of the porous medium, $C_{p}$ is the specific heat at constant pressure, $\nu$ is the kinematic viscosity, $\sigma$ is the electrical conductivity of the fluid, $N$ is the components of micro rotation or angular velocity whose rotation is in the direction of the $x-y$ plane, and $j, \gamma$ and $k_{1}^{*}$ are the microinertia per unit mass, spin gradient viscosity and vortex viscosity respectively, $q_{r}$ is the radiation heat flux, $\beta_{t}$ and $\beta_{c}$ are the coefficient of thermal expansion and concentration expansion respectively. The spin gradient viscosity $\gamma$, which defines the relationship between the coefficient of viscosity and microinertia are as follows:

$$
\gamma=\mu\left(1+\frac{K}{2}\right) j
$$

in which $K=\frac{k_{1}^{*}}{\mu}(\mu>0)$ is the material parameter. Here all the material constants, $\gamma, \mu, k, j$ are non-negative and we take $j=\frac{\nu}{b}$ as a reference length. The appropriate physical boundary conditions for the problem under study are given by

$$
\begin{gathered}
u=u_{w}=b x, v=0, N=-n \frac{\partial u}{\partial y} \text { at } y=0 \\
-k \frac{\partial T}{\partial y}=q_{w}=D_{0}\left(\frac{x}{l}\right)^{2},-D \frac{\partial c}{\partial y}=m_{w}=D_{1}\left(\frac{x}{l}\right)^{2} \text { at } y=0 \\
u \rightarrow 0, N \rightarrow 0 \text { as } y \rightarrow \infty \\
T \rightarrow T_{\infty}, C \rightarrow C_{\infty} \text { as } y \rightarrow \infty .
\end{gathered}
$$

Here $l$ is the characteristic length, $T_{w}$ is the wall temperature of the fluid and $T_{\infty}$ is the temperature of the fluid far away from the sheet, $C_{w}$ is the wall concentration of the solute and $C_{\infty}$ is the concentration of the solute far away from the sheet, $D_{o}, D_{1}$ are constant and $k=k_{\infty}(1+\epsilon \theta(\eta))$, Chiam [4]; $n$ is a constant such that $0 \leq n \leq 1$. The case when $n=0$, is called strong concentration, which indicates $N=0$ near the wall represents concentrated particle flows in which the micro-elements close to the wall surface are unable to rotate, see Mathur and Jena [10]. The case when $n=\frac{1}{2}$ indicates the vanishing of antisymmetric part of the stress tensor and denotes weak concentrations where as $n=1$ is used for modelling of turbulent boundary layer flows, Ahmadi [1]. It is worth mentioning that $k=0$ describes the classical Navier-Stokes equation for 
a viscous and incompressible fluid. The non-uniform heat source/sink is given by

$$
q^{\prime \prime \prime}=\frac{k U_{w}}{x \nu}\left[A^{*}\left(T_{w}-T_{\infty}\right) f^{\prime}+B^{*}\left(T-T_{\infty}\right)\right],
$$

where $A^{*}$ and $B^{*}$ are the coefficients of space and temperature - dependent heat source/sink, respectively. The case $A *>0$ and $B *>0$ corresponds to internal heat generation while $A^{*}<0$ and $B^{*}<0$ corresponds to internal heat absorption. We use the following similarity variables and dimensionless steam function to transform equation (2) and (3):

$$
U=b x f^{\prime}(\eta), v=\sqrt{b \nu} f(\eta), \eta=\sqrt{\frac{b}{\nu}} y, N=b x\left(\frac{b}{\nu}\right)^{\frac{1}{2}} g(\eta) .
$$

Substituting (12) into equation (2) and (3), we have

$$
\begin{gathered}
f^{\prime 2}-f f^{\prime \prime}=(1+K) f^{\prime \prime \prime}-D a^{-1} f^{\prime}-\alpha f^{\prime 2}+K g^{\prime}-H a^{2} f^{\prime}+G_{t}, \theta+G_{c} \phi, \\
f^{\prime} g-f g^{\prime}=\left(1+\frac{K}{2}\right) g^{\prime \prime}-K\left(2 g+f^{\prime \prime}\right),
\end{gathered}
$$

where $\alpha=\frac{C_{b} \varphi x}{\sqrt{k}}$ is local inertia coefficient parameter, $D a^{-1}=\frac{\varphi \nu}{k b}$ is inverse Darcy number, $H a=\sqrt{\frac{\sigma}{\rho b}} B_{0}$ is the Hartmann number, $G_{t}=\frac{\left.g \beta_{t}\left(T-T_{\infty}\right)\right)}{b^{2}}$ is local temperature Grashof number, $G_{c}=\frac{g \beta_{t}\left(C-C_{\infty}\right)}{b^{2}}$ is local concentration Grashof number and $K=\frac{k_{1}^{*}}{\mu}$ is material parameter. The appropriate boundary conditions (7) and (9) now become as follows:

$$
\begin{aligned}
f(\eta)= & 0, f^{\prime}(\eta)=1, g(\eta)=-n f^{\prime \prime}(\eta) \text { at } \eta=0, \\
& f^{\prime}(\eta) \rightarrow 0, g(\infty) \rightarrow 0 \text { as } \eta \rightarrow \infty,
\end{aligned}
$$

where $n=\frac{1}{2}$, we can take $g(\eta)=-\frac{1}{2} f^{\prime \prime}(\eta)$. So combining (13) and(14), we will reduce to a single non-linear ordinary differential equation as

$$
f^{\prime 2}-f f^{\prime \prime}=\left(1+\frac{K}{2}\right) f^{\prime \prime \prime}-D a^{-1} f^{\prime}-\alpha f^{\prime 2}-H a^{2} f^{\prime}+G_{t} \theta+G_{c} \phi .
$$

Subject to the appropriate boundary conditions

$$
f(\eta)=0, f^{\prime}(\eta)=1 \text { at } \eta=0, f(\eta) \rightarrow 0 \text { as } \eta \rightarrow \infty,
$$

following Rosseland approximation (Brewstar [3]) the radiative heat flux $q_{r}$ is modeled as

$$
q_{r}=-\frac{4 \sigma^{*}}{3 k^{*}} \frac{\partial T^{4}}{\partial y} .
$$


Here $\sigma^{*}$ is the Stefan-Boltzman constant and $k^{*}$ is the mean absorption coefficient. Assuming that the difference in temperature within the flow are such that $T^{4}$ can be expressed as a linear combination of the temperature, we expand $T^{4}$ in Taylor's series about $T_{\infty}$ as follows:

$$
T^{4}=T_{\infty}^{4}+4 T_{\infty}^{3}\left(T-T_{\infty}\right)+6 T_{\infty}^{2}\left(T-T_{\infty}\right)^{2}+\ldots,
$$

and neglecting higher order terms beyond the first degree in $\left(T-T_{\infty}\right)$, we have

$$
T^{4} \approx-3 T_{\infty}^{4}+4 T_{\infty}^{3} T .
$$

Differentiating equation (18) with respect to $y$ and using equation (20), we obtain

$$
\frac{\partial q_{r}}{\partial y}=-\frac{16 T_{\infty}^{3} \sigma^{*}}{3 k^{*}} \frac{\partial^{2} T}{\partial y^{2}} .
$$

Substituting equation (21) into equation (4), we have

$$
u \frac{\partial T}{\partial x}+v \frac{\partial T}{\partial y}=\frac{1}{\rho C_{p}}\left(K+16 \frac{T_{\infty}^{3} \sigma^{*}}{3 k^{*}}\right) \frac{\partial^{2} T}{\partial y^{2}}+\frac{\sigma B_{0}^{2}}{\rho C_{p}} u^{2}+\frac{q^{\prime \prime \prime}}{\rho C_{p}}+\frac{\mu}{\rho C_{p}}\left(\frac{\partial u}{\partial y}\right)^{2} .
$$

The thermal boundary conditions for solving (22) depend on the type of heating process considered. Now the non-dimensional temperature $\theta(\eta)$ and concentration $\phi(\eta)$ are define (in PHF case) as

$$
\theta(\eta)=\frac{T-T_{\infty}}{T_{w}-T_{\infty}}, \phi(\eta)=\frac{C-C_{\infty}}{C_{w}-C_{\infty}},
$$

where

$$
\begin{aligned}
& T-T_{\infty}=\frac{D_{0}}{K_{\infty}}\left(\frac{x}{l}\right)^{2} \sqrt{\frac{\nu}{b}} \theta(\eta) \text { and } T_{w}-T_{\infty}=\frac{D_{0}}{K_{\infty}}\left(\frac{x}{l}\right)^{2} \sqrt{\frac{\nu}{b}} \\
& C-C_{\infty}=\frac{D_{1}}{D}\left(\frac{x}{l}\right)^{2} \sqrt{\frac{\nu}{b}} \phi(\eta) \text { and } C_{w}-C_{\infty}=\frac{D_{1}}{D}\left(\frac{x}{l}\right)^{2} \sqrt{\frac{\nu}{b}} .
\end{aligned}
$$

Using equation (23) into equation (22), yields

$$
\begin{aligned}
\left(1+N_{r}+\epsilon \theta\right) \theta^{\prime \prime}+ & \operatorname{Pr}\left(f \theta^{\prime}-f^{\prime} \theta\right)+\epsilon \theta^{2}+\operatorname{Pr} H a^{2} E_{s} f^{\prime} 2 \\
& +(1+\epsilon \theta)\left(A^{*} f^{\prime}+B^{*} \theta\right)+\operatorname{Pr} E_{s} f^{\prime \prime}=0 .
\end{aligned}
$$

Subject to the boundary conditions

$$
\theta(\eta)=\frac{1}{1+\epsilon} a t \eta=0, \theta(\eta) \rightarrow 0 \text { as } \eta \rightarrow \infty
$$


using equation (23) in equation (5), we have

$$
\phi(\eta)=S c\left(\phi^{\prime} f-2 \phi f^{\prime}\right)=0 .
$$

And the corresponding thermal boundary conditions are:

$$
\phi(\eta)=\frac{1}{1+\epsilon} a t \eta=0, \phi(\eta) \rightarrow 0 \text { as } \eta \rightarrow \infty,
$$

where $N_{r}=\frac{16 T^{3} \sigma^{*}}{3 k^{*} K_{\infty}}$ is thermal radiation parameter, $\operatorname{Pr}=\frac{\mu C_{p}}{1 K_{\infty}}$ is Prandth number, $E_{s}=E_{c} K_{\infty} \sqrt{\frac{b}{\nu}}$ is the scaled Ecket number, $E_{c}=\frac{b^{2} l^{2}}{D_{0} C_{p}}$ is the Eckert number and $S_{c}=\frac{\nu}{b}$ is the Schmidt number.

\section{Method of Solution}

Equation (16), (26) and (28) are highly coupled non-linear ordinary differential equation and since $\epsilon<<1$, we assume a perturbation of this form

$$
\begin{gathered}
f=1+\epsilon f_{1}+\epsilon^{2} f_{2}, \\
\theta=-\frac{1}{1+\epsilon}+\epsilon \theta_{1}+\epsilon^{2} \theta_{2}, \\
\phi=-\frac{1}{1+\epsilon}+\epsilon \phi_{1}+\epsilon^{2} \phi_{2},
\end{gathered}
$$

where $f_{0}=1, \theta_{0}=-\frac{1}{1+\epsilon}, \phi_{0}=-\frac{1}{1+\epsilon}$. Invoking equation (30) - (32) into equation (16), (26) and (28) and neglecting terms of $O\left(\epsilon^{3}\right)$ and higher, we have the following sets of equations

$$
\begin{gathered}
\left(1+\frac{K}{2}\right) f_{1}^{\prime \prime \prime}+f_{1}^{\prime \prime}-\left(D a^{-1}+H a^{2}\right) f_{1}^{\prime}=-G_{t} \theta_{1}-G_{c} \phi_{1}, \\
\left(1+N_{r}\right) \theta_{1}^{\prime \prime}+\operatorname{Pr} \theta_{1}^{\prime}+B^{*} \theta_{1}=-\frac{B^{*}}{(1+\epsilon)^{2}}, \\
\phi_{1}^{\prime \prime}+S c \phi_{1}^{\prime}=0, \\
\left(1+\frac{K}{2}\right) f_{2}^{\prime \prime \prime}+f_{2}^{\prime \prime}-\left(D a^{-1}+H a^{2}\right) f_{2}^{\prime}=f_{1} f_{1}^{\prime \prime 2}-\alpha f^{\prime 2}-G_{t} \theta_{2}-G_{c} \phi_{2}, \\
\left(1+N_{r}\right) \theta_{2}^{\prime \prime}+\operatorname{Pr} \theta_{2}^{\prime}+B \theta_{2}=-\frac{1}{1+\epsilon} \theta_{1}^{\prime \prime}-\operatorname{Pr} f_{1} \theta_{1}^{\prime}-\frac{2}{1+\epsilon} \operatorname{Pr} f_{1}^{\prime 2} \\
-\operatorname{Pr} H a^{2} E_{s} f_{1}^{\prime 2}-A^{*} f^{\prime 2}+\frac{2}{1+\epsilon} \theta_{1},
\end{gathered}
$$




$$
\phi_{2}^{\prime \prime}+S c \phi_{2}^{\prime}=-S c \phi_{1}^{\prime} f_{1}-\frac{2}{1+\epsilon} S c f_{1}^{\prime 2},
$$

Solving equation (33) - (38) with the boundary conditions to obtain the expression for velocity, temperature and concentration distributions as

$$
\begin{aligned}
f(\eta)= & 1+\epsilon\left(A_{3}+A_{4} e^{-m_{2} \eta}+A_{6} e^{-m_{1} \eta}+A_{7} e^{-S c \eta}\right)+\epsilon^{2}\left(A_{25}+A_{26} e^{-m_{2} \eta}\right. \\
& +A_{27} \eta e^{-m_{2} \eta}+A_{28} e^{-2 m_{2} \eta}+A_{29} e^{-\left(m_{1}+m_{2}\right) \eta}+A_{30} e^{-\left(S c+m_{2}\right) \eta}+ \\
& A_{31} e^{-m_{1} \eta}+A_{32} e^{-2 m_{1} \eta}+A_{33} e^{-\left(S c+m_{1}\right) \eta} \\
+ & A_{34} e^{-S c \eta}+A_{35} e^{-2 S c \eta} \\
\theta(\eta)= & -\frac{1}{1+\epsilon}+\epsilon\left(A_{2} e^{-m_{1} \eta}-\frac{1}{(1+\epsilon)^{2}}\right)+\epsilon^{2}\left(A_{17} e^{-m_{1} \eta}\right. \\
& +A_{18} \eta e^{-m_{1} \eta}+A_{19} e^{-\left(m_{1}+m_{2}\right) \eta}+A_{20} e^{-2 m_{1} \eta}+A_{21} e^{-\left(S c+m_{2}\right) \eta} \\
& +A_{22} e^{-2 m_{2} \eta}+A_{23} e^{-\left(S c+m_{2}\right) \eta}+A_{24} e^{-2 S c \eta}, \\
\phi(\eta)= & -\frac{1}{1+\epsilon}+\epsilon A_{1} e^{-S c \eta}+\epsilon^{2}\left(A_{9} e^{-S c \eta}+A_{10} \eta e^{-S c \eta}+A_{11} e^{-\left(S c+m_{2}\right) \eta}\right. \\
& +A_{12} e^{-\left(S c+m_{1}\right) \eta}+A_{13} e^{-2 S c \eta}+A_{14} e^{-2 m_{2} \eta} \\
& +A_{15} e^{-\left(m_{1}+m_{2}\right) \eta}+A_{16} e^{-2 m_{1} \eta} .
\end{aligned}
$$

Here:

$$
\begin{aligned}
& m_{1}=\frac{P r+\sqrt{P r^{2}-4\left(1+N_{r}\right) B^{*}}}{2\left(1+N_{r}\right)} \\
& m_{2}=\frac{1+\sqrt{1+4\left(1+\frac{K}{2}\right)\left(D a^{-1}+H a^{2}\right)}}{2\left(1+\frac{K}{2}\right)} \\
& A_{1}=-\frac{1}{1+\epsilon} \\
& A_{2}=-\frac{1}{1+\epsilon}+\frac{1}{(1+\epsilon)^{2}} \\
& A_{6}=\frac{-G_{t} A_{2}}{-\left(1+\frac{K}{2}\right) m_{1}^{3}+m_{1}^{2}+\left(D a^{-1}+H a^{2}\right) m_{1}} \\
& A_{7}=\frac{-G_{c} A_{1}}{-\left(1+\frac{K}{2}\right) S c_{1}^{3}+S c_{1}^{2}+\left(D a^{-1}+H a^{2}\right) S c} \\
& A_{4}=-\frac{1+S c A_{7}+m_{1} A_{6}}{m_{2}} \\
& A_{3}=-\left(A_{4}+A_{6}+A_{7}\right)
\end{aligned}
$$




$$
\begin{aligned}
& A_{10}=\frac{S c^{2} A_{1} A_{3}}{3 S c} \\
& A_{11}=\frac{(1+\epsilon) S c^{2} A_{1} A_{4}-4 S c^{2} m_{2} A_{4} A_{7}}{(1+\epsilon)\left(\left(S c+m_{2}\right)^{2}-S c\left(S c+m_{2}\right)\right)} \\
& A_{12}=\frac{(1+\epsilon) S c^{2} A_{1} A_{6}-4 S c^{2} m_{1} A_{6} A_{7}}{(1+\epsilon)\left(\left(S c+m_{1}\right)^{2}-S c\left(S c+m_{1}\right)\right)} \\
& A_{13}=\frac{(1+\epsilon) S c^{2} A_{1} A_{7}-2 S c^{3} A_{7}^{2}}{\left.(1+\epsilon) 2 S c^{2}\right)} \\
& A_{14}=\frac{-2 S c m_{2}^{2} A_{4}^{2}}{4 m_{1}^{2}-2 S c m_{2}} \\
& A_{15}=-\frac{4 S c m_{1} m_{2} A_{4} A_{6}}{(1+\epsilon)\left(\left(m_{1}+m_{2}\right)^{2}-S c\left(m_{1}+m_{2}\right)\right)} \\
& A_{16}=-\frac{2 S c m_{1}^{2} A_{6}^{2}}{(1+\epsilon)\left(4 m_{1}^{2}-S c m_{1}\right)} \\
& A_{9}=-\left(\frac{1}{1+\epsilon}+A_{11}+A_{12}+A_{13}+A_{14}+A_{15}+A_{16}\right) \\
& A_{18}=\frac{A_{2} m_{1}^{2}+(1+\epsilon) \operatorname{Pr} A_{2} m_{1} A_{3}}{1+\epsilon} \\
& A_{19}=\frac{(1+\epsilon) \operatorname{Pr} A_{2} m_{1} A_{4}-2 \operatorname{Pr}+(1+\epsilon) \operatorname{Pr} H a^{2} E_{s}-(1+\epsilon) A^{*}}{(1+\epsilon)\left(\left(1+N_{r}\right)\left(m_{1}+m_{2}\right)^{2}-\operatorname{Pr}\left(m_{1}+m_{2}\right)+B^{*}\right)} \\
& A_{20}=\frac{(1+\epsilon) \operatorname{Pr}_{2} m_{1} A_{6}-2 \operatorname{Prm}_{1}^{2} A_{6}^{2}+(1+\epsilon) \operatorname{PrHa}^{2} E_{s} m_{1}^{2} A_{6}^{2}-(1+\epsilon) A^{*} m_{1}^{2} A_{6}^{2}}{(1+\epsilon)\left(\left(1+N_{r}\right) 4 m_{1}^{2}-2 \operatorname{Prm}_{1}+B^{*}\right)} \\
& A_{21}=\frac{(1+\epsilon) \operatorname{Pr} A_{2} m_{1} A_{6}-4 \operatorname{Pr} S c m_{1} A_{6} A_{7}+(1+\epsilon) 2 \operatorname{PrHa} E_{s} m_{1} A_{6} A_{7}-2(1+\epsilon) A^{*} m_{1} A_{6} A_{7}}{(1+\epsilon)\left(\left(1+N_{r}\right)\left(S c+m_{1}\right)^{2}-\operatorname{Pr}\left(S c+m_{1}\right)+B^{*}\right)} \\
& A_{22}=\frac{-2 \operatorname{Prm}_{1}^{2} A_{4}^{2}-(1+\epsilon) \operatorname{PrHa}^{2} E_{s} m_{2}^{2} A_{4}^{2}+(1+\epsilon) A^{*} m_{2}^{2} A_{4}^{2}}{(1+\epsilon)\left(\left(1+N_{r}\right) 4 m_{2}^{2}-2 \operatorname{Prm}_{2}+B^{*}\right)} \\
& A_{23}=\frac{-2 m_{2} A_{4} S c A_{7}\left(2 \operatorname{Pr}+(1+\epsilon) \operatorname{Pr} H a^{2} E_{s}-(1+\epsilon) A^{*}\right)}{(1+\epsilon)\left(\left(1+N_{r}\right)\left(m_{2}+S c\right)^{2}-\operatorname{Pr}\left(m_{2}+S c\right)+B^{*}\right)} \\
& A_{24}=\frac{-S c^{2} A_{7}^{2}\left(2 \operatorname{Pr}+(1+\epsilon) \operatorname{Pr} H a^{2} E_{s}-(1+\epsilon) A^{*}\right)}{(1+\epsilon)\left(\left(1+N_{r}\right) 4 S c^{2}-\operatorname{Pr} S c+B^{*}\right)} \\
& A_{17}=-\left(\frac{1}{1+\epsilon}+A_{19}+A_{20}+A_{21}+A_{22}+A_{23}+A_{24}\right) \\
& A_{27}=\frac{-A_{3} A_{4} m_{2}^{2}}{\left(1+\frac{K}{2}\right) 3 m_{2}^{2}-2 m_{2}-\left(D a^{-1}+H a^{2}\right)} \\
& A_{28}=\frac{A_{4}^{2} m_{2}^{2}+(\alpha-1)\left(m_{2}^{2} A_{4}^{2}+G_{t} A_{22}+G_{c} A_{14}\right)}{\left(1+\frac{K}{2}\right) 8 m_{2}^{3}-4 m_{2}^{2}-\left(D a^{-1}+H a^{2}\right) m_{2}} \\
& A_{29}=\frac{A_{4} A_{6}\left(m_{2}^{2}+m_{1}^{2}+2(\alpha-1) m_{1} m_{2}\right)+G_{t} A_{19}+G_{c} A_{15}}{\left(1+\frac{K}{2}\right)\left(m_{1}+m_{2}\right)^{3}-\left(m_{1}+m_{2}\right)^{2}-\left(D a^{-1}+H a^{2}\right)\left(m_{1}+m_{2}\right)} \\
& A_{30}=\frac{A_{4} A_{7}\left(m_{2}^{2}-S c^{2}-2(\alpha-1) m_{2} S c\right)+G_{t} A_{23}+G_{c} A_{11}}{\left(1+\frac{K}{2}\right)\left(S c+m_{2}\right)^{3}-\left(S c 1+m_{2}\right)^{2}-\left(D a^{-1}+H a^{2}\right)\left(S c+m_{2}\right)}
\end{aligned}
$$




$$
\begin{aligned}
& A_{31}=\frac{A_{6} A_{3} m_{1}^{2}-G_{t} A_{17}}{\left(1+\frac{K}{2}\right) m_{1}^{3}-m_{1}^{2}-\left(D a^{-1}+H a^{2}\right) m_{1}} \\
& A_{32}=\frac{A_{6}^{2} m_{1}^{2}(1+(\alpha-1))+G_{t} A_{20}+G_{c} A_{16}}{\left(1+\frac{K}{2}\right) 8 m_{1}^{3}+4 m_{1}^{2}+2\left(D a^{-1}+H a^{2}\right) m_{1}} \\
& A_{33}=\frac{A_{6} A_{7}\left(m_{1}^{2}+S c^{2}+2(\alpha-1) m_{1}\right)+G_{t} A_{21}+G_{c} A_{12}}{\left(1+\frac{K}{2}\right)\left(S c+m_{1}\right)^{3}-\left(S c 1+m_{1}\right)^{2}+\left(D a^{-1}-H a^{2}\right)\left(S c+m_{1}\right)} \\
& A_{34}=\frac{A_{7} A_{3} S c^{2}+G_{c} A_{9}}{\left(1+\frac{K}{2}\right) S c^{3}-S c^{2}+\left(D a^{-1}-H a^{2}\right) S c} \\
& A_{35}=\frac{A_{7}^{2} S c^{2}(1+(\alpha-1))+G_{t} A_{24}+G_{c} A_{13}}{\left(1+\frac{K}{2}\right) 8 S c^{3}+4 S c^{2}+\left(D a^{-1}+H a^{2}\right) 2 S c} \\
& A_{26}=\frac{1+2 m_{2} A_{28}+\left(m_{1}+m_{2}\right) A 29+\left(S c+m_{2}\right) A_{30}+m_{1} A_{31}+2 m_{1} A_{32}+\left(S c+m_{1}\right) A_{33}+S c A_{34}+2 S c A^{*}}{m_{2}} \\
& A_{25}=-\left(A_{26}+A_{28}+A_{29}+A_{30}+A_{31}+A_{32}+A_{34}+A_{35}\right) .
\end{aligned}
$$

The physical quantity of most interest in science and engineering is the skin-friction coefficient $C_{f}$, the Nusselt number $\mathrm{Nu}$ and Sherwood number Sh which are defined by the following relations

$$
C_{f}=\frac{2 \tau_{w}}{\rho U_{w}^{2}},
$$

and the skin-friction on the plate $\tau(w)$ is given by

$$
\tau_{w}=\left(\left(\mu+k_{1}^{*}\right) \frac{\partial u}{\partial y}\right)_{y=0} .
$$

Substituting equation (12) in (42) and using (43), we have

$$
C_{f} R e_{x}^{\frac{1}{2}}=(1+K) f^{\prime \prime}(0),
$$

where $R e_{x}=\frac{U_{w} x}{\nu}$ is the local Reynolds number.

The local Nusselt number is given by

$$
N_{u}=\frac{\left(\frac{\partial T}{\partial y}\right)_{y=0}}{\left(T_{w}-T_{\infty}\right) \sqrt{\frac{b}{\nu}}}=-\theta^{\prime}(0)
$$

and the local Sherwood number is given by

$$
S_{h}=\frac{\left(\frac{\partial C}{\partial y}\right)_{y=0}}{\left(C_{w}-C_{\infty}\right) \sqrt{\frac{b}{\nu}}}=-\phi^{\prime}(0) .
$$




\section{Results and Discussion}

The formulation of the hydromagnetic boundary layer flow, heat and mass transfer characteristics on steady two-dimensional flow with uniform magnetic field in the presence of thermal radiation of a micropolar fluid over a porous medium has been performed in the preceding sections. In order to understand the physical situation of the problem and hence the manifestation of the various parameters entering the problem, we have carried out the numerical calculations for distribution of velocity, temperature and concentration across the boundary layer for different values of the parameters. In this present study we have chosen $A^{*}=0.01, B^{*}=0.01, K=0.2, \mathrm{Es}=0.05, \epsilon=0.01$, while Ha, $G_{t}, G_{c}, D a^{-1}, \alpha$, $\mathrm{Nr}, \mathrm{Pr}$ and $\mathrm{Sc}$ are varied over a range which are listed in figures legends. Figure 2 shows the behaviour of velocity profile for different values of Hartmann number Ha. It is well known that the Hartmann number represents the importance of magnetic field on the flow. As depicted from Figure 2, when the Hartmann number increases, the velocity profile decreases. This is due to the fact that the introduction of transverse magnetic field normal to the flow direction has a tendency to create a drag due to Lorentz force and hence results in retarding the velocity profile. Thus when the Hartmann number increases, the Lorentz force also increases due to which velocity profile decreases. From Figure 3 and Figure 4 , it is observed that the effect of increasing the value of the thermal Grashof number $G_{r}$ and concentration Grashof number $G_{c}$ is to increase the velocity profile. Figure 5 illustrates the variation of velocity profile with $\eta$ for various value of inverse Darcy number. The plot shows that velocity profile decreases with increase in the inverse Darcy number which shows the effect of increasing inverse Darcy number is to decrease the velocity profile. Similar effects are seen in case of increasing inertia coefficient parameter $\alpha$ as shown in Figure 6. Figure 7 represents the temperature profiles for various values of thermal radiation parameter $\mathrm{Nr}$ in the boundary layer. This figure shows that the effect of thermal radiation is to enhance heat transfer because of the fact that thermal boundary layer thickness increases with increase in the thermal radiation. Thus it is pointed out that the radiation should be minimized to have the cooling process at a faster rate. Figure 8 illustrates the variation of temperature profile for various values of Prandtl number. It is seen that the temperature decreases with increasing the values of Prandtl number in the boundary layer which is evident that temperature in the boundary layer falls very quickly for large value of the Prandtl number because of the fact that thickness of the boundary layer decreases with increase in the value of the Prandtl number. Figure9 shows the variation of temperature profile with $\eta$ for various values of inverse 
Darcy number $D a^{-1}$. It depicts that temperature increases with increase in the value of inverse Darcy number which is due to the fact that obstruction on the fluid motion is produced by the presence of porous medium which generates heat and thereby temperature increases in the thermal boundary layer. Figure 10 shows that temperature increases with increase in the inertia coefficient parameter. Figure 11 is the plot of concentration distribution for various values of Schmidt number in the boundary layer. It is illustrated from the figure that the concentration decreases with increase in the value of Schmidt number. This is due to the fact that increase in Schmidt number causes thinning of the solutal boundary layer thickness. It should be noted that the present results are in excellent agreement with the results reported by Abel etal(2008) and Dulal and Sewli (2009). Numerical values of the skin-friction coefficient $f^{\prime \prime}(0)$, the wall temperature $\theta(0)$ and the wall solutal concentration $\phi(0)$ are tabulated in table1 for different values of material parameter K, Hartmann number Ha, Prandt number Pr and inverse Darcy number $D a^{-1}$. The tabular data shows that magnetic field, Prandt number, inverse Darcy number and material parameter reduce the skin-friction coefficient, whereas reverse trend is seen by increasing the values of Es, $G_{t}, G_{c}$ and Nr. It is further observed that wall temperature increases with increase in $\mathrm{K}, \mathrm{Ha}, \mathrm{Es}, \mathrm{Nr}$ and $D a^{-1}$ whereas opposite effect is seen with increasing the value of $G_{t}, G_{c}$, and Pr. The effect of increasing the values of $\mathrm{K}, \mathrm{Ha}, \operatorname{Pr}, D a^{-1}$ has the tendency to increase wall solutal concentration but the other parameters like Es, $G_{t}, G_{c}$ and $\mathrm{Nr}$ have the effect of decreasing $\phi(0)$. Table 2 depicts the numerical values of coefficient of skin friction $C_{f}$, Nusselt number $\mathrm{Nu}$ and Sherwood number $S_{h}$ for different values of $\mathrm{Ha}, \mathrm{Nr}$ and $\mathrm{Pr}$. The tabular data shows that Nusselt number and Sherwood number decreases with increasing in Hartmann number, Nusselt number increases as radiation parameter increases while the effect of increasing the value of Prandth number is to decrease the skin friction coefficient and mass transfer rate but increase heat transfer rate which is in excellent agreement with Sewli and Dulal [16] and Srinivasachanya and Ramreddy [17].

\section{Conclusion}

In this work, we have studied steady boundary layer flow and heat transfer of a micropolar fluid-saturated porous medium past an impermeable stretching sheet with magnetic field and thermal radiation effects using the DarcyBrinkman Forchermer model. The resulting partial differential equations which describe the problem, are transformed into ordinary differential equations by 
using similarity transformations. The resulted non-linear ordinary differential equations are solved by perturbation technique. Following conclusions can be drawn from the results obtained:

* Velocity decreases with increase in Hartmann number, inverse Darcy number and inertia coefficient parameter but reverse trend is seen by increase thermal Grashof number Gt and solutal Grashof number Gc,

* Temperature increases with increase in the value of the thermal radiation, inverse Darcy number and inertia coefficient parameter whereas it decreases with increase in the value of the Prandt number,

* Concentration decreases with increase in the value of the Schmidt number.

The results are presented as velocity, microrotation, temperature and concentration profiles in Figures 2-11 and Tables 1-2 below:

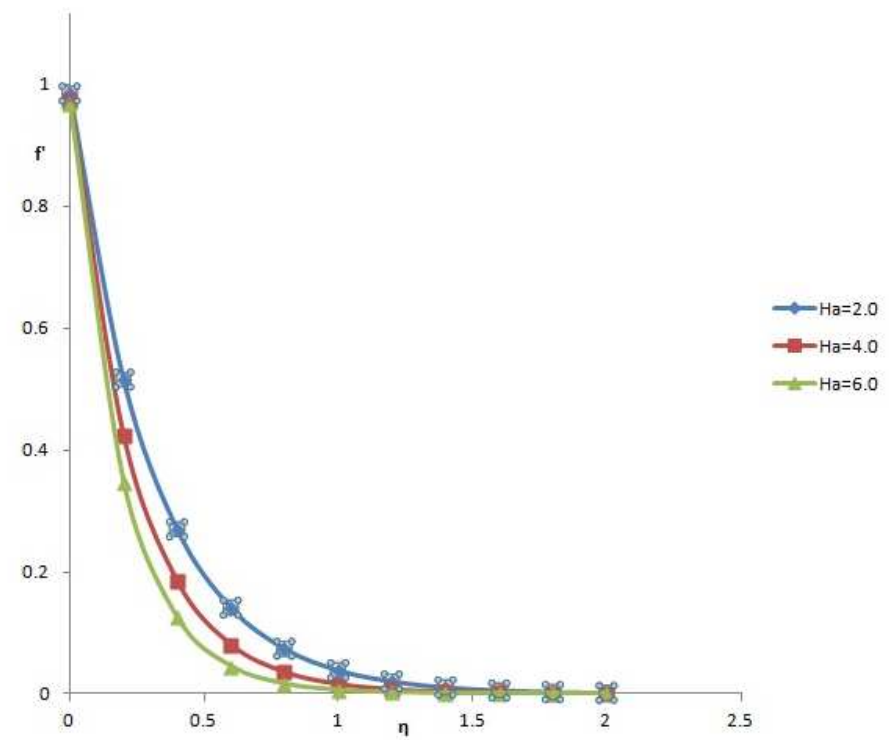

Figure 2: Variation of $f^{\prime}$ for different values of Ha. 


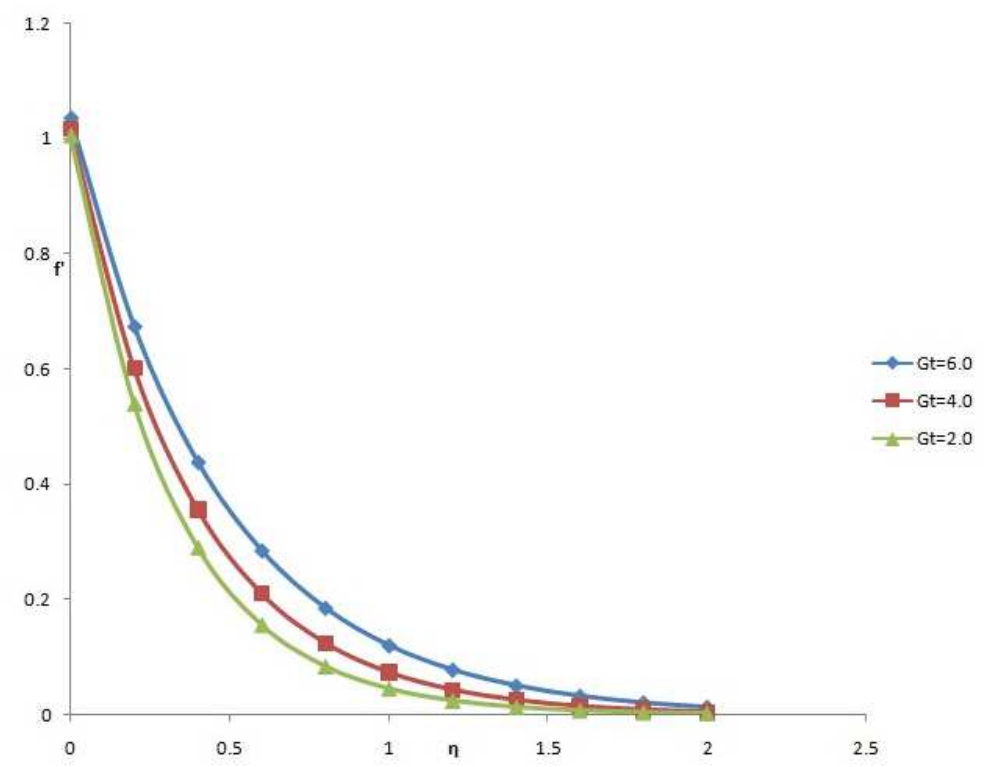

Figure 3: Variation of $f^{\prime}$ for different values of Gt.

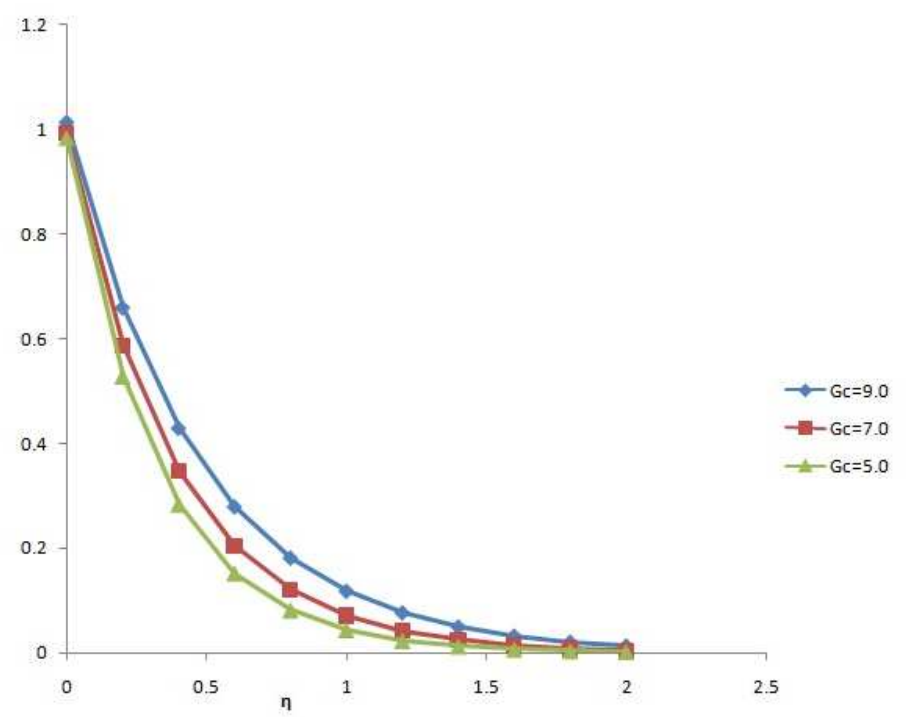

Figure 4: Variation of $f^{\prime}$ for different values of Gc. 


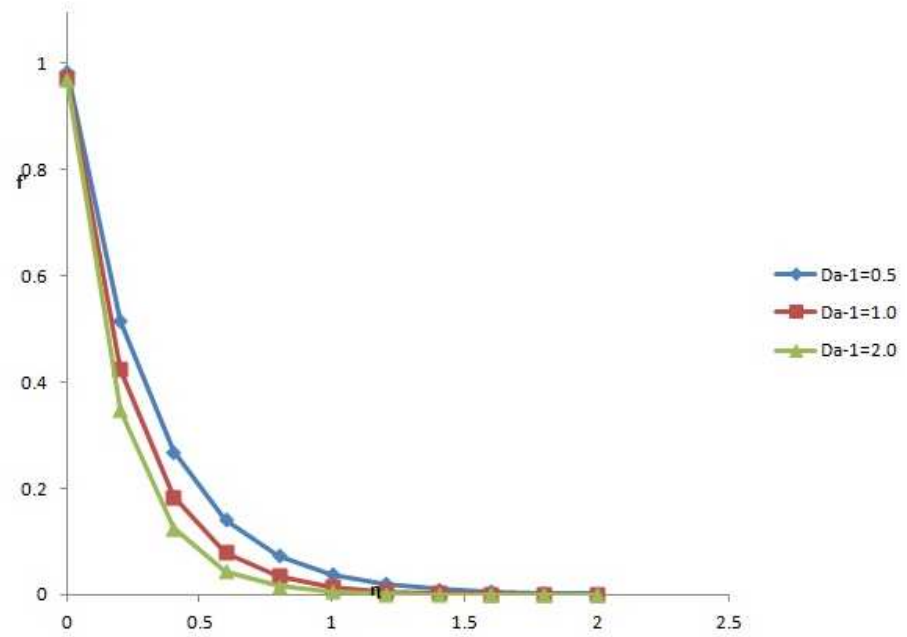

Figure 5: Variation of $f^{\prime}$ for different values of $D a^{-1}$

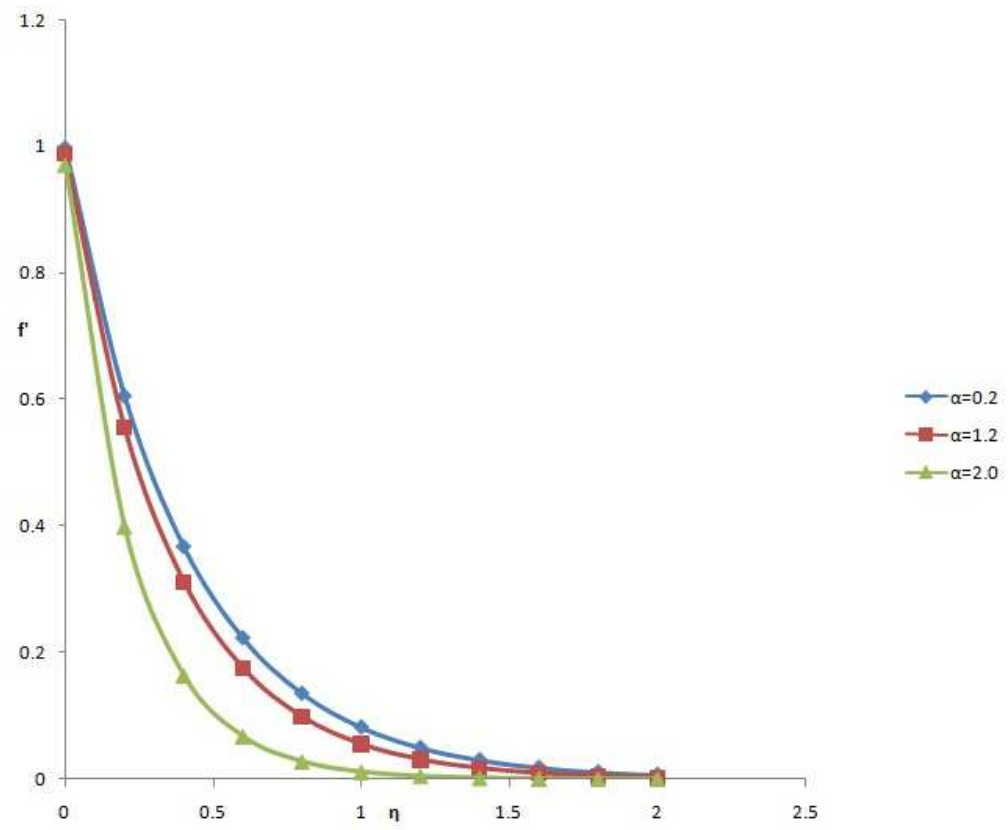

Figure 6: Variation of $f^{\prime}$ for different values of $\alpha$. 


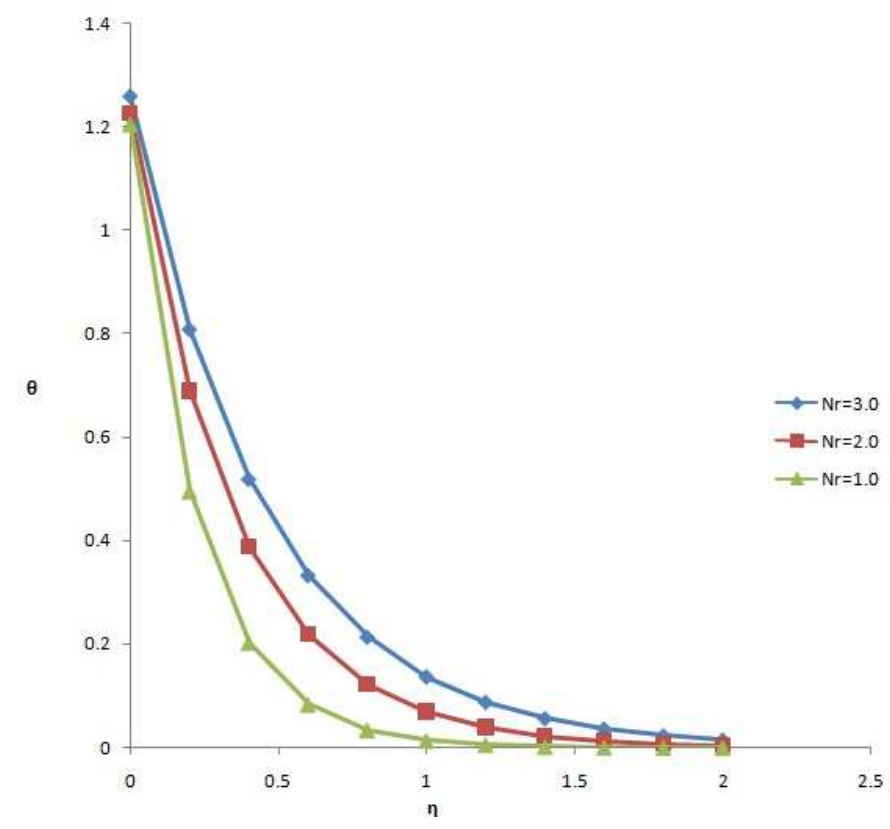

Figure 7: Variation of $\theta$ for different values of $\mathrm{Nr}$.

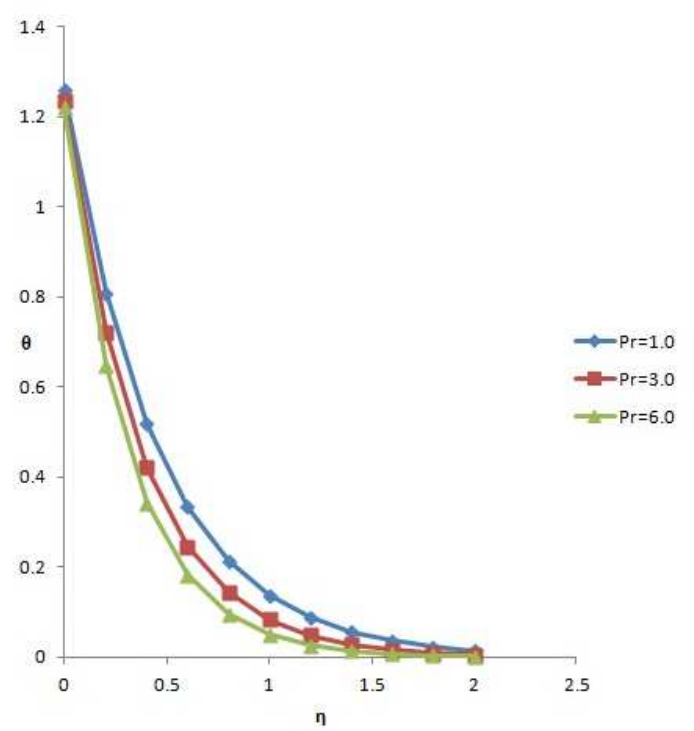

Figure 8: Variation of $\theta$ for different values of $\mathrm{Pr}$. 


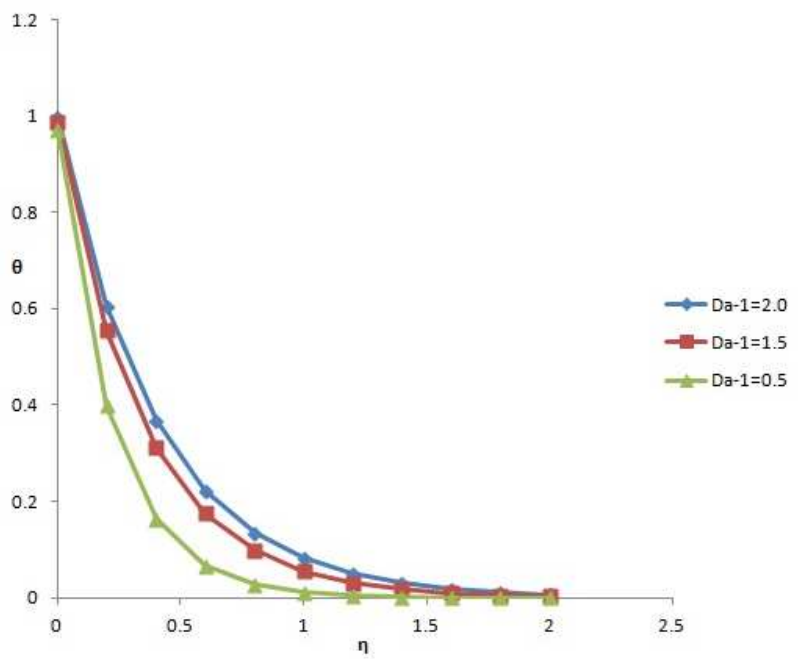

Figure 9: Variation of $\theta$ for different values of $D a^{-1}$

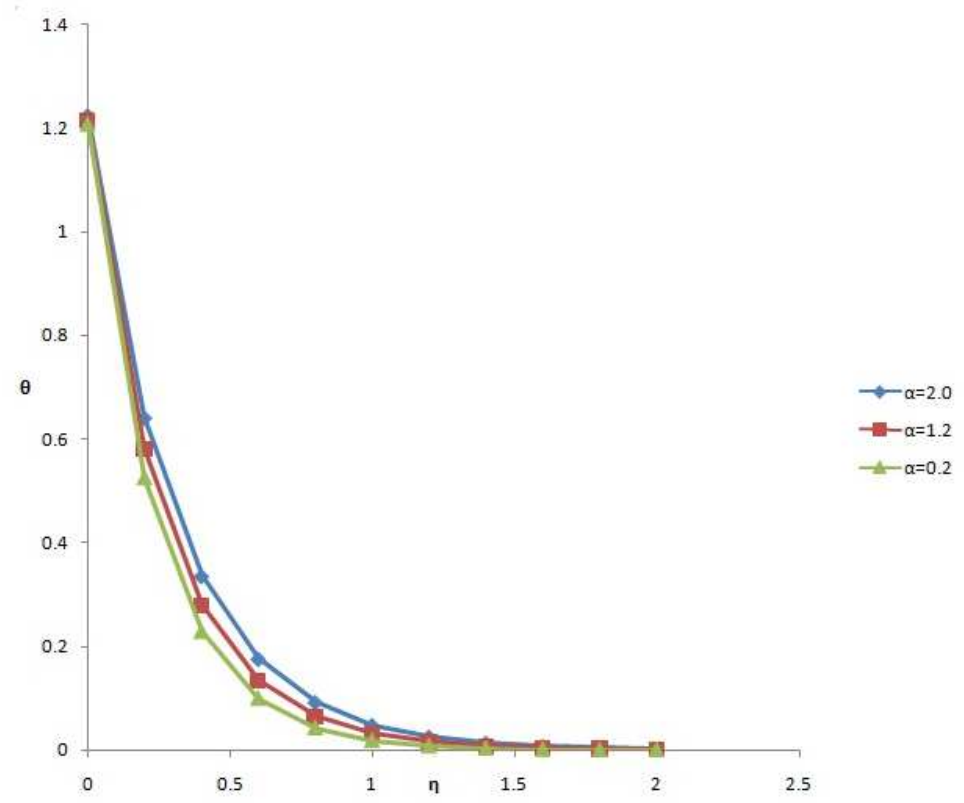

Figure 10: Variation of $\theta$ for different values of $\alpha$. 


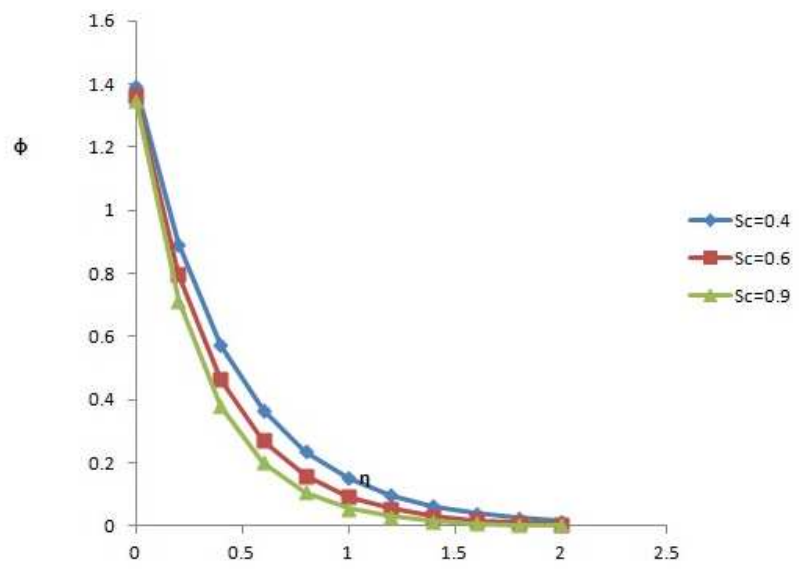

Figure 11: Variation of $\phi$ for different values of Sc.

\begin{tabular}{|c|c|c|c|c|c|c|c|c|c|c|}
\hline $\mathrm{K}$ & $\mathrm{Ha}$ & $\mathrm{Es}$ & $G_{t}$ & $G_{c}$ & $\mathrm{Nr}$ & $\mathrm{Pr}$ & $\mathrm{Da}^{-1}$ & $f^{\prime \prime}(0)$ & $\theta(0)$ & $\phi(0)$ \\
\hline 1.0 & 0.1 & 0.05 & 5.0 & 4.0 & 3.5 & 3.0 & 0.5 & 1.60434 & 0.70583 & 1.31521 \\
2.0 & 0.1 & 0.05 & 5.0 & 4.0 & 3.5 & 3.0 & 0.5 & 1.32150 & 0.71848 & 1.35369 \\
0.2 & 1.0 & 0.05 & 5.0 & 4.0 & 3.5 & 3.0 & 0.5 & 1.61046 & 0.74224 & 1.36108 \\
0.2 & 2.0 & 0.05 & 5.0 & 4.0 & 3.5 & 3.0 & 0.5 & 0.79668 & 0.87770 & 1.53207 \\
0.2 & 0.1 & 0.05 & 5.0 & 4.0 & 3.5 & 3.0 & 0.5 & 1.97707 & 0.71402 & 1.31493 \\
0.2 & 0.1 & 0.25 & 5.0 & 4.0 & 3.5 & 3.0 & 0.5 & 1.97922 & 0.71551 & 1.31356 \\
0.2 & 0.1 & 0.05 & 6.0 & 4.0 & 3.5 & 3.0 & 0.5 & 2.12904 & 0.70855 & 1.30228 \\
0.2 & 0.1 & 0.05 & 8.0 & 4.0 & 3.5 & 3.0 & 0.5 & 2.42051 & 0.69794 & 1.28087 \\
0.2 & 0.1 & 0.05 & 5.0 & 5.0 & 3.5 & 3.0 & 0.5 & 2.30513 & 0.69968 & 1.27993 \\
0.2 & 0.1 & 0.05 & 5.0 & 7.0 & 3.5 & 3.0 & 0.5 & 2.89612 & 0.65795 & 1.20833 \\
0.2 & 0.1 & 0.05 & 5.0 & 4.0 & 5.0 & 3.0 & 0.5 & 2.18918 & 0.83756 & 1.26950 \\
0.2 & 0.1 & 0.05 & 5.0 & 4.0 & 6.0 & 3.0 & 0.5 & 2.28906 & 0.89762 & 1.25801 \\
0.2 & 0.1 & 0.05 & 5.0 & 4.0 & 3.5 & 4.0 & 0.5 & 1.82115 & 0.60911 & 1.31143 \\
0.2 & 0.1 & 0.05 & 5.0 & 4.0 & 3.5 & 6.0 & 0.5 & 1.65949 & 0.50298 & 1.32867 \\
0.2 & 0.1 & 0.05 & 5.0 & 4.0 & 3.5 & 3.0 & 0.6 & 1.91600 & 0.69905 & 1.30365 \\
0.2 & 0.1 & 0.05 & 5.0 & 4.0 & 3.5 & 3.0 & 2.0 & 1.39126 & 0.73491 & 1.40513 \\
\hline
\end{tabular}

Table 1: Values of $f^{\prime \prime}(0), \theta(0), \phi(0)$ for different values of $\mathrm{K}$, Ha, Es, $G_{t}, G_{c}, \operatorname{Pr}, \mathrm{Nr}$ and $D a^{-1}$ when $A=0.01, B=0.01$ and $\epsilon=0.01$ 


\begin{tabular}{|c|c|c|c|c|c|}
\hline $\mathrm{Ha}$ & $\mathrm{Nr}$ & $\mathrm{Pr}$ & $C_{f}$ & $N_{u}$ & $S_{h}$ \\
\hline 0.0 & 3.5 & 3.0 & 1.97683 & 0.34286 & 0.47826 \\
1.0 & 3.5 & 3.0 & 1.62968 & 0.24105 & 0.46217 \\
2.0 & 3.5 & 3.0 & 0.80966 & 0.15098 & 0.41151 \\
0.1 & 5.0 & 3.0 & 2.21928 & 0.12037 & \\
0.1 & 6.0 & 3.0 & 2.32961 & 0.18692 & \\
0.1 & 7.0 & 3.0 & 2.42161 & 0.24324 & \\
0.1 & 3.5 & 4.0 & 1.84265 & 0.31832 & 0.20160 \\
0.1 & 3.5 & 6.0 & 1.67848 & 0.41641 & 0.11923 \\
0.1 & 3.5 & 8.0 & 1.58619 & 0.71502 & 0.01902 \\
\hline
\end{tabular}

Table 2: Values of $C_{f}, N_{u}$ and Sh with different values of $\mathrm{Ha}, \mathrm{Nr}$ and Pr when $A=0.01, B=0.01, K=1.0, \mathrm{Es}=0.05, G_{t}=5.0, G_{c}=4.0$, $D a^{-1}=0.5, \epsilon=0.01$ and $\alpha=0.1$

\section{References}

[1] G. Ahmadi, Self-similar solution of incompressible micropolar boundary layer flow over a semi-infinite plate, Int. J. Eng. Sci., 14 (1976), 639-646.

[2] M. Bayomi, A.S. Chamkha and E.M. Khedr, MHD flow of a micropolar fluid past a stretched permeable surface with heat generation or absorption, Non-analysis, Modeling and Control, 14 (2009), 27-40.

[3] M.Q. Brewster, Thermal Radiation Transfer Properties, John Wiley and Sons, 1972.

[4] T.C. Chiam, Heat transfer in a fluid with variable thermal conductivity over sretching sheet, Acta Mechanica 129 (1998), 63-72.

[5] N.T. Eldabe and M.E. Ouat, Chebyshew finite difference method for heat and mass transfer in hydromagnetic flow of a micropolar fluid past a stretching surface with Ohmic heating and viscous dissipation, Appl. Math. Comput., 177 (2006), 561-571.

[6] N.A. Keelson and A. Desseaux, Effects of surface condition on flow of a micropolar fluid driven by a porous stretching sheet, Int. J. Eng. Sci, 39 (2001), 1881-1897.

[7] M.A. Mahmoud, Thermal radiation effects on MHD flow of a micropolar fluid over a stretching surface with variable thermal conductivity, Physica A, 375 (2007), 401-410. 
[8] R.A. Mohammed and S.M Abo-Dahab, Influence of chemical reaction and thermal radiation on the heat and mass transfer in MHD micropolar flow over a vertical moving porous plate in a porous medium with heat generation, International Journal of Thermal Sciences, 48 (2009), 1800-1813.

[9] R.A. Mohammed, S.M. Abo-Dahab, and T.A. Nofa, Thermal radiation and MHD effects on free convective flow of a polar fluid through a porous medium in the presence of internal heat generation and chemical reaction, Mathematical problems in Engineering, 27 (2007), 10-11.

[10] M.N. Mathur and S.K. Jena, Similarity solutions for laminar free convection flow of a thermo-micropolar fluid past a non-isothermal flat plate, Int. J. Eng. Sci. 19 (1981), 1437.

[11] A.C. Magdy, Free convection flow of conducting micropolar fluid with thermal relaxation including heat sources, Journal of Applied Mathematics, 2 (2005), 271-292.

[12] A.D. Rehbi, A.A. Tariq, A.S. Benbella and A.A. Mahoud, Unsteady natural convection heat transfer of micropolar fluid over a vertical surface with constant heat, flux, Turkish J. Eng. Env. Sci. 31 (2007), 225-233.

[13] M.A. Rahmann and T. Sultan, Radiative heat transfer flow of micropolar fluid with variable heat flux in a porous medium, Nonlinear Analysis modeling and control, 13 (2008), 71-87.

[14] A. Reena and U.S. Rana, Linear stability of thermo solutal convection in a micropolar fluid saturating a porous medium, Intern. Journal of Application and Applied Mathematics, 4 (2009), 62-87.

[15] A. Sunil, A. Sharma, P.K. Bharti and R.G. Shandi, Effect of rotation on a layer of micropolar ferromagnetic fluid heated from below saturating a porous medium, International Journal of Engineering Science, 44 (2006), 683-698.

[16] C. Sewli and P. Dulal, Heat and mass transfer in MHD non-Darcian flow of a micropolar fluid over a stretching sheet embedded in a porous media with non- uniform heat source and thermal radiation, Commun Nonlinear Sci. Simulation, 15 (2010), 1843-1857.

[17] D. Srinivasachanya and Ch. Ramreddy, Soret and Dufour effect on mixed convection in a non-Darcy porous medium saturated with micropolar fluid, Nonlinear Analysis Modelling and Control, 16 (2011), 100-115. 
